\title{
Characterization of Randomly Branched Polydisperse Polymers
}

\author{
Hideomi Matsuda, Izumi Yamada, and Shigetaka Kuroiwa \\ Department of Chemical Engineering, Faculty of Textile \\ Science, Shinshu University, Ueda 386, Japan.
}

(Received February 26, 1976)

\begin{abstract}
A new method for characterizing randomly branched polydisperse polymers is proposed. Measurements of sedimentation velocity and diffusion of the branched polymer at the $\theta$ point of the corresponding linear polymer give two apparent molecular weight distribution curves. From these curves the real molecular weight distribution curve can be obtained without any effect of branching, and the branching parameter distribution curve can be calculated. This new method has been applied to polystyrene-divinylbenzene copolymers; the results show that the method is very simple and useful for the characterization of branched polymers.

KEY WORDS Branch / Polymer Characterization / Sedimentation / Diffusion / Molecular Weight Distribution / Dilute Solution /
\end{abstract}

It is well known that the dilute solution properties of a branched polymer are influenced by its molecular weight distribution and branching distribution, thus making the characterization of a branched polymer very difficult. The following experimental methed for characterizing branched polymers has been proposed. A polydisperse sample is fractionated by the ordinary fractionation method, and the branching parameter of each fraction, which is approximately monodisperse, is determined by viscosity or light scattering measurements. Experimental data have been reported by Thurmond and Zimm $^{1}$ for styrene-divinylbenzene copolymer, by Guillet ${ }^{2}$ for low density polyethylene, and by Blachford and Robertson ${ }^{3}$ for butadienestyrene copolymer. Theoretical calculations of solution properties have been attempted by many workers. ${ }^{4-6}$ Kilb reported a method of determining the branching parameter of a randomly branched polydisperse polymer by viscosity measurements. ${ }^{7 /}$ Moreover, Kurata, et al., studied the intrinsic viscosity and sedimentation constant of randomly branched polystyrene. ${ }^{8}$ Kamada and Sato studied the dilute solution properties of randomly branched poly(methyl methacrylate)..$^{9,10}$ These studies, however, did not show how to obtain the molecular weight and branching parameter distributions. Since gel-permeation chromatography (GPC) has been used for polymer characterization, studies of branched polymers have also been advanced by the GPC technique. ${ }^{11-13}$ Tung proposed a method to obtain molecular weight and branching parameter distributions of randomly branched polydisperse polymers by combining GPC and sedimentation velocity measurements. ${ }^{14,15}$ In any case, in order to get molecular weight and branching parameter distributions separately, solution properties, which are influenced directly by the hydrodynamic radius of the polymer in solution and by which the molecular weight distribution of polymers can be obtained, should be measured: for example, the sedimentation constant, diffusion constant, and retention volume of GPC. However, there are some unsolved problems in the absolute measurement of the molecular weight distribution by GPC, and therefore the sedimentation velocity and diffusion measurements are expected to be most useful to obtain the molecular weight and branching parameter distributions separately for randomly branched polydisperse polymers.

In this paper, we propose a new method, which combines sedimentation velocity and diffusion measurements in dilute polymer solutions, for getting the molecular weight and branching parameter distributions of a randomly branched polydisperse polymer, and we report some experimental results. 


\section{THEORETICAL}

As the theory for the characterization of a randomly branched polydisperse polymer is complex, we first consider a randomly branched monodisperse polymer, which cannot really be prepared, and then apply the theory to a randomly branched polydisperse polymer.

Molecular Weight and Branching Parameter of $a$

Randomly Branched Monodisperse Polymer

For a monodisperse linear polymer at the $\theta$ temperature, the relation between the molecular weight $M$ and the hydrodynamic radius $R$ is given by

$$
M=K_{\mathrm{r}} R^{2}
$$

where $K_{\mathrm{r}}$ is a constant. Whether the sample is linear or not, if the partial specific volume of the branched polymer in solution is assumed to be equal to that of a linear polymer, then the sedimentation constant $S$ is given by

$$
S=K_{\mathrm{S}} M / R
$$

where $K_{\mathrm{S}}$ is a constant. Equation 2 is an alternative expression of the well-known Svedberg's equation. ${ }^{16}$ Substituting eq 1 into eq 2 , we have

$$
S=K_{\mathrm{S}} K_{\mathrm{r}}^{0.5} M^{0.5}
$$

As eq 1 is applicable only to a linear polymer at the $\theta$ temperature, eq 3 corresponds to the following relation between the molecular weight and the sedimentation constant of a linear polymer at the $\theta$ temperature:

$$
S=K_{\mathrm{S}}{ }^{\prime} M^{0.5}
$$

If the sedimentation constant of a branched polymer is measured at the $\theta$ temperature of the corresponding linear polymer and the molecular weight is calculated by eq 3 or 4 , then the molecular weight of the branched polymer is

$$
M_{\mathrm{S}}=S^{2} / K_{\mathrm{S}}{ }^{2} K_{\mathrm{r}}=S^{2} / K_{\mathrm{S}}{ }^{2}
$$

The molecular weight $M_{\mathrm{S}}$ calculated by eq 5 is the apparent molecular weight, for eq 3 and 4 are valid only for a linear polymer at the $\theta$ temperature. The difference between the apparent molecular weight $M_{\mathrm{S}}$ and the real one $M$ depends upon the degree of branching. On the other hand, the relation between the diffusion constant $D$ and the hydrodynamic radius
$R$ is, regardless whether the sample is branched or not, given by the equation

$$
D=K_{\mathrm{D}} / R
$$

where $K_{\mathrm{D}}$ is a constant. This equation is an alternative expression of the Einstein-Stokes' equation. Substituting eq 1 into eq 6 , we have the equation

$$
D=K_{\mathrm{D}} K_{\mathrm{r}}^{0.5} / M^{0.5}
$$

Equation 7 corresponds to the following equation for a linear polymer at the $\theta$ temperature:

$$
D=K_{\mathrm{D}}{ }^{\prime} M^{-0.5}
$$

If the diffusion constant of a branched polymer is measured at the $\theta$ temperature of the corresponding linear polymer and if the molecular weight is calculated by eq 7 or 8 , then an apparent molecular weight $M_{\mathrm{D}}$ should be obtained as

$$
M_{\mathrm{D}}=K_{\mathrm{D}}{ }^{2} K_{\mathrm{r}} / D^{2}=K_{\mathrm{D}}{ }^{\prime 2} / D^{2}
$$

As eq 2 and 6 are valid also for a branched polymer, the real molecular weight of a branched polymer is expressed as

$$
M=K_{\mathrm{D}} S / K_{\mathrm{S}} D
$$

Then, the substituting of eq 5 and 9 into eq 10 gives the real molecular weight:

$$
M=\left(M_{\mathrm{D}} \cdot M_{\mathrm{S}}\right)^{0.5}
$$

Therefore, if the sedimentation constant and the diffusion constant of a branched polymer are measured at the $\theta$ temperature for the corresponding linear polymer, and if the apparent molecular weights $M_{\mathrm{S}}$ and $M_{\mathrm{D}}$ are calculated by eq 4 and 8 for the linear polymer, then the real molecular weight of the branched polymer is obtained by eq 11. Among the apparent molecular weights $M_{\mathrm{D}}, M_{\mathrm{S}}$, and the real molecular weight $M$, we have the relation

$$
M_{\mathrm{D}}<M<M_{\mathrm{S}}
$$

For the linear polymer, we have the relation

$$
M_{\mathrm{D}}=M=M_{\mathrm{S}}
$$

Equation 11 can be used to obtain the molecular weight distribution of a randomly branched polydisperse polymer.

Stockmayer and Fixman define a branching 
parameter $h$ as ${ }^{4}$

$$
h=R_{\mathrm{b}} / R_{1}
$$

where $R_{\mathrm{b}}$ and $R_{1}$ are the hydrodynamic raddi of branched and linear polymers, respectively, in solutions having the same molecular weight. Substituting eq 11 into eq 1 we obtain

$$
R_{1}{ }^{2}=\left(M_{\mathrm{D}} \cdot M_{\mathrm{S}}\right)^{0.5} / K_{\mathrm{r}}
$$

This expression relates the hydrodynamic radius of a linear polymer to the apparent molecular weights $M_{\mathrm{D}}$ and $M_{\mathrm{S}}$, which can be obtained experimentally. On the other hand, from eq 6 and 7 , the hydrodynamic radius of a branched polymer is expressed as

$$
R_{\mathrm{b}}{ }^{2}=M_{\mathrm{D}} / K_{\mathrm{r}}
$$

Substituting eq 15 and 16 into eq 14 we have

$$
h^{2}=\left(M_{\mathrm{D}} / M_{\mathrm{S}}\right)^{0.5}
$$

Equation 17 shows that the branching parameter $h$ can be calculated from the apparent molecular weights $M_{\mathrm{D}}$ and $M_{\mathrm{S}}$ of a branched polymer.

Molecular Weight and branching Parameter Distribution Curves of a Randomly Branched Polydisperse Polymer

Our method needs to be generalized for polydisperse polymer as follows. Using the method proposed to obtain the molecular weight distribution for a linear polymer, ${ }^{17,18}$ we obtain integral distribution curves of the apparent molecular weights $M_{\mathrm{D}}$ and $M_{\mathrm{S}}$ of the branched polymer from diffusion and sedimentation velocity measurements at the $\theta$ temperature of the corresponding linear polymer. Then, using eq 11 , we can calculate the real molecular weight $M$ for each set of points with the same integral values on the integral distribution curves of the apparent molecular weights $W\left(M_{\mathrm{S}}\right)$ and $W\left(M_{\mathrm{D}}\right)$. The integral distribution curve of the real molecular weight $W(M)$ is obtained by joining a series of points of the real molecular weights.

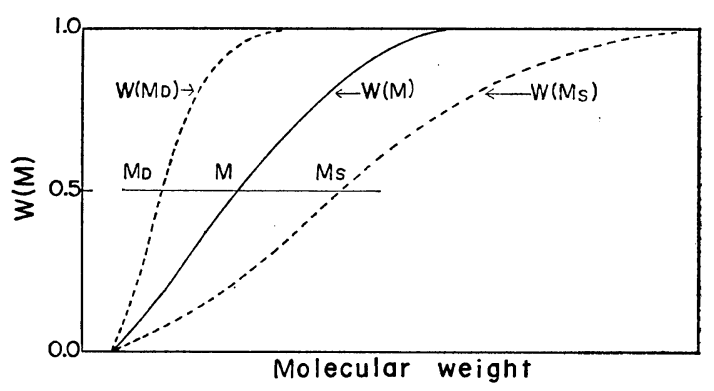

Figure 1. Calculation method of molecular weight distribution curve (MWDC) of branched polymer: $W\left(M_{\mathrm{D}}\right)$, apparent MWDC obtaind from diffusion method; $W\left(M_{\mathrm{S}}\right)$, apparent MWDC obtained from sedimentation velocity method; $W(M)$, real MWDC calculated by $M=\left(M_{\mathrm{D}} \cdot M_{\mathrm{S}}\right)^{0.5}$.

(See Figure 1.) Similarly, the distribution curve of the branching parameter can be obtained by eq 17. Thus, we can obtain the molecular weight and branching parameter distribution curves of a randomly branched polydisperse polymer.

\section{EXPERIMENTAL}

\section{Materials}

Two kinds of branched polymers, PSB-1 and PSB-2 (styrene-divinylbenzene copolymer), and a corresponding linear polymer, PSL (polystyrene), were used in the present study. Styrene-divinylbenzene copolymers were polymerized in our laboratory according to the method of Thurmond and Zimm. ${ }^{1}$ Polymerization conditions are shown in Table I. Polymerization mixtures were dissolved into a mixture of dioxane and acetone (1:1 by volume), which is a very good solvent for branched polymers. The resulting solution was added into an excess amount of methanol to obtain a polymer precipitate. The precipitate was filtered off and resolved in MEK. The solution was

Table I. Polymerization conditions of branched polymers

\begin{tabular}{ccccccc}
\hline & $\begin{array}{c}\text { Styrene, } \\
\mathrm{m} l\end{array}$ & $\begin{array}{c}\text { Divinyl benzene, } \\
\mathrm{m} l\end{array}$ & $\begin{array}{c}\mathrm{AIBN}^{\mathrm{a}}, \\
\mathrm{g}\end{array}$ & $\begin{array}{c}\text { Temp, } \\
{ }^{\circ} \mathrm{C}\end{array}$ & $\begin{array}{c}\text { Time, } \\
\text { hours }\end{array}$ & $\begin{array}{c}\text { Conv, } \\
\%\end{array}$ \\
\hline PSB-1 & 60 & 0.055 & 0.046 & 70 & 7.0 & 31 \\
PSB-2 & 80 & 0.045 & 0.038 & 55 & 19.0 & 15 \\
\hline
\end{tabular}

a $\mathrm{AIBN}=$ azobisisobutyronitrile. 
added into methanol and the precipitate was filtered, then dried under reduced pressure at $50^{\circ} \mathrm{C}$ for 3 days. The number-average and weight-averge molecular weights were determined by osmotic pressure and light-scattering measurements. In order to compare the results with those of a branched polymer, a linear polystyrene sample was used; this was distributed by Professor M. Takeda of Tokyo College of Science for use as a standard sample in Japan. The number-average and weight-average molecular weights of this sample were measured in our laboratory.

Measurement of $\theta$ Point of Branched Polystyrene in Cyclohexane

In our theory, all experiments have to be carried out at the $\theta$ point of the corresponding linear polymer, and thus the $\theta$ point of the branched polymer must be determined. If the difference of the $\theta$ points between the linear and branched polymers is very large, our theory cannot be used. Samples, which were fractionated from a part of PSB-2, were dissolved into cyclohexane at a concentration about $3 \mathrm{~g} / 100 \mathrm{~m} l$. in glass ampoules; then they were immersed in a thermostat at a constant temperature. After one night the solution separated into two liquid phases and the concentration of polymer in each phase was measured. The $\theta$ point of branched polystyrene in cyclohexane was obtained from these data according to Flory's method. ${ }^{19}$ As is shown in the Result section, the $\theta$ point of this system is $34.2{ }^{\circ} \mathrm{C}$, which is very close to that of the corresponding linear polymer.

\section{Sedimentation Velocity Measurements}

Sedimentation velocity measurements were carried out at $35^{\circ} \mathrm{C}$ in cyclohexane using a Hitachi Ultracentrifuge with Schlieren optics. All measurements were made at $43,700 \mathrm{rpm}$ and at four concentrations. Apparent molecular weight distribution curves were obtained by the method proposed for a linear polymer with corrections of diffusion and concentration; ${ }^{17}$ the correction of the pressure effect on the sedimentation constant was neglected.

\section{Diffusion Measurements}

Diffusion measurements were carried out in cyclohexane at $35^{\circ} \mathrm{C}$ using a Hitachi HTB-type electrophoresis apparatus with Schlierene optics. A modified Neurath cell was used. Apparent molecular weight distribution curves were obtained according to Gralén's method for a linear polymer. ${ }^{20}$

\section{Viscosity Measurements}

The intrinsic viscosity of the branched polymer was measured in cyclohexane at $35^{\circ} \mathrm{C}$, and that of the linear polymer having the same molecular weight was calculated by the equation ${ }^{21}$

$$
[\eta]=7.48 \times 10^{-4} M^{0.5}, \quad(100 \mathrm{ml} / \mathrm{g})
$$

\section{RESULTS}

Phase separation curves of branched poly-

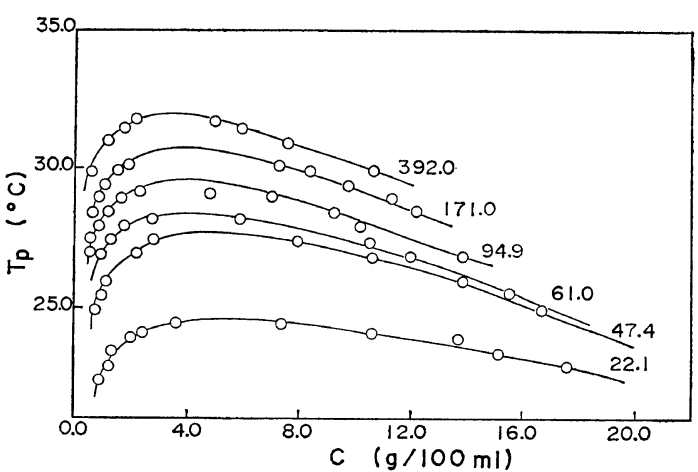

Figure 2. Relation between phase separation temperature and concentration of branched polymer in cyclohexane. The figures show molecular weight in $10^{-4}$.

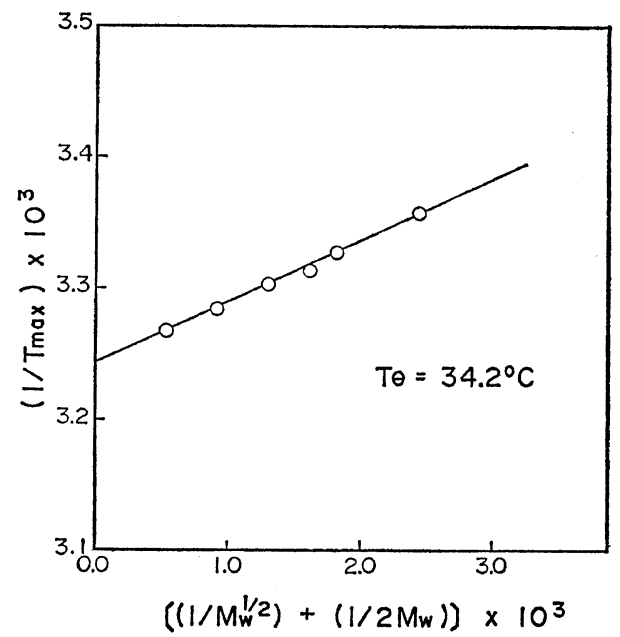

Figure 3. Relation between $T_{\max }$ and the molecular weight of branched polymer in cyclohexane. 


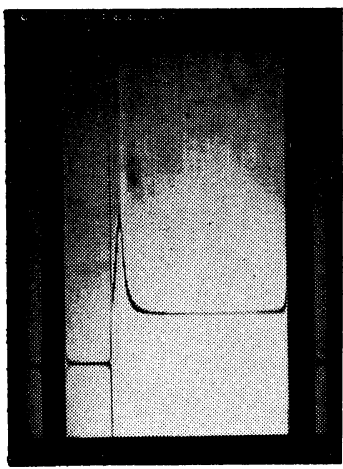

$780 \mathrm{sec}$

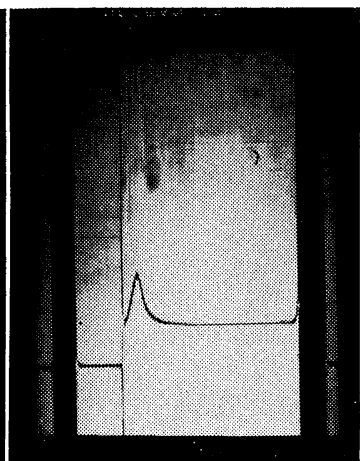

$1140 \mathrm{sec}$

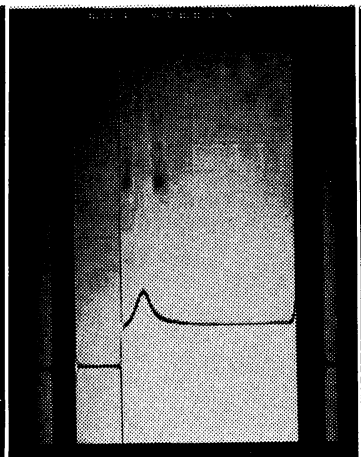

$1400 \mathrm{sec}$

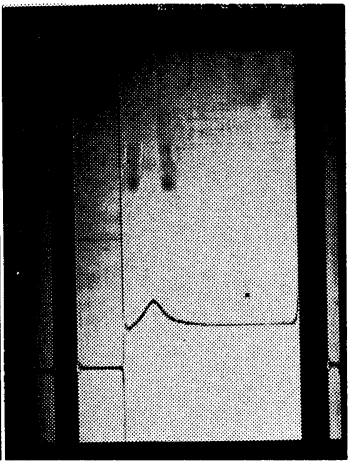

$1760 \mathrm{sec}$

Figure 4. Examples of photographs of sedimentation velocity measurements. The figures show sedimentation time in sec. Sample: PSB-1; concn, $0.6 \mathrm{~g} / 100 \mathrm{ml}$.

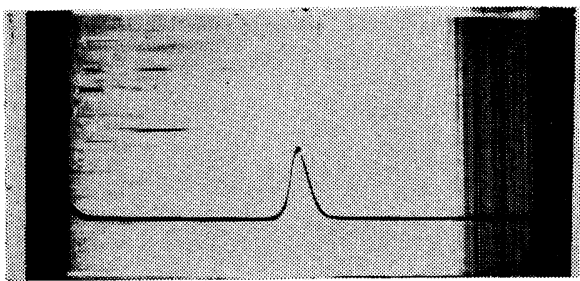

$4.0 \mathrm{hr}$

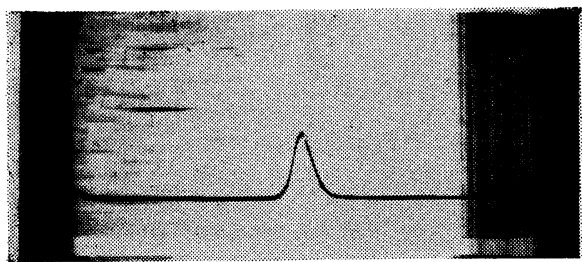

$8.0 \mathrm{hr}$

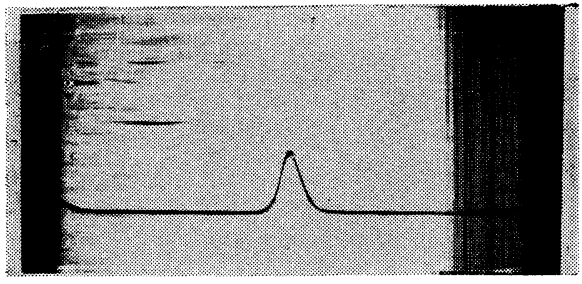

$12.0 \mathrm{hr}$

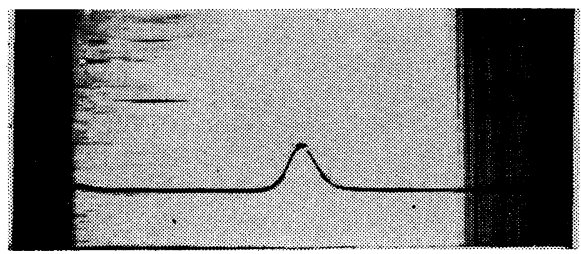

$16.0 \mathrm{hr}$

Figure 5. Examples of diffusion curves. The figures show diffusion time in hours. Sample: PSB-1; concn, $0.8 \mathrm{~g} / 100 \mathrm{~m} l$.

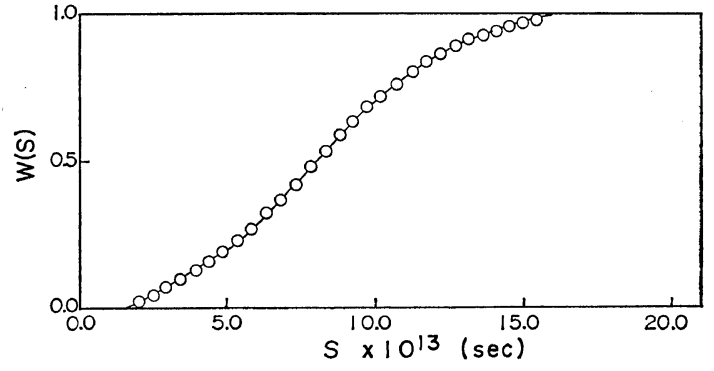

Figure 6. Integral weight distribution curve of sedimentation constant of PSB-1.

styrene in cyclohexane are show in Figure 2. As the samples were not monodisperse, the maximum points of these curves might deviate a little from the critical temperatures. However, the temperature at the maximum point $T_{\max }$ was approximated as the critical temperature and the $\theta$ temperature was estimated from the $T_{\max }$ by using the Flory method, as shown in Figure 3. The $\theta$ temperature of this system is $34.2^{\circ} \mathrm{C}$ and is almost the same as that of linear polystyrene in cyclohexane. Therefore, our method was practically applicable for this branched polymer. As described in the Experimental section, all the results were obtained in cyclohexane at $35^{\circ} \mathrm{C}$. Examples of photographs of sedimentation and diffusion measurements are shown in Figures 4 and 5. Examples of integral distribution curves of sedimentation and diffusion constants are shown in Figures 6 and 7, respectively. From these curves, the apparent molecular weight distribution curves are calculated by the following equations of a linear polymer: 


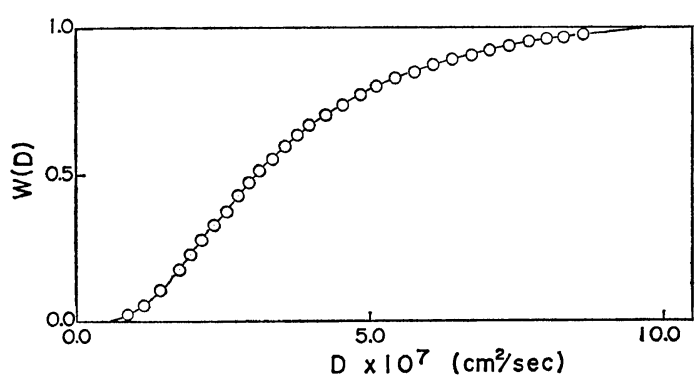

Figure 7. Integral weight distribution curve of diffusion constant of PSB-1.

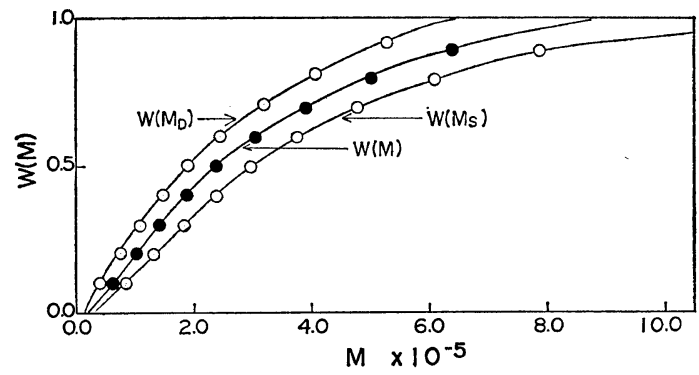

Figure 8. Integral weight distribution curves of molecular weight of PSB-1.

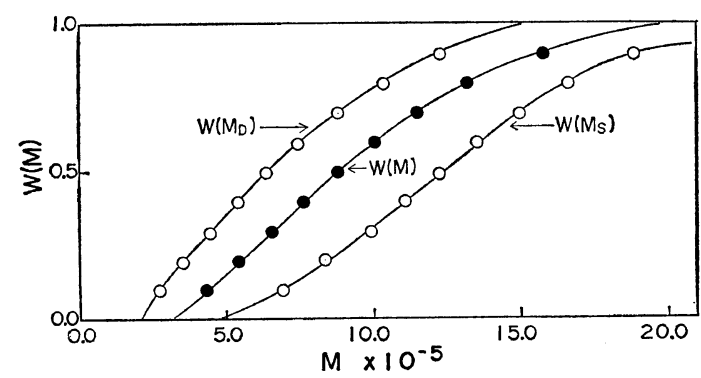

Figure 9. Integral weight distribution curves of molecular weight of PSB-2.

$$
\begin{gathered}
S=1.48 \times 10^{-15} M^{0.5}, \quad(\mathrm{sec})^{22} \\
D=1.43 \times 10^{-4} M^{-0.5}, \quad\left(\mathrm{~cm}^{2} / \mathrm{sec}\right)^{23}
\end{gathered}
$$

Integral distribution curves of the apparent molecular weight are shown together with integral distribution curves of the real molecular weight of branched polymers calculated by eq 11 in Figures 8 and 9 . In order to examine the experimental accuracy, the integral molecular weight distribution curves of linear polystyrene obtained by the sedimentation velocity and diffusion method are shown in Figure 10. Both distribution curves in Figure 10 are very close to each other, within experimental errors. Therefore Figure 10 indicates that our experiments were accurately carried out. The branching parameter distribution curves calculated by eq 17 are shown in Figure 11. The number-average and weight-average molecular weights, $M_{n}$ and $M_{w}$, and the cubes of the weight average of branching parameters, $\left(h_{w}\right)^{3}$ calculated from the distribution curves are shown in Table II. The same table also lists the observed values of $M_{n}$, $M_{w}$, and $\left(h_{w}\right)^{3}=[\eta]_{\mathrm{b}} /[\eta]_{1}$, where $[\eta]_{1}$ and $[\eta]_{\mathrm{b}}$ are

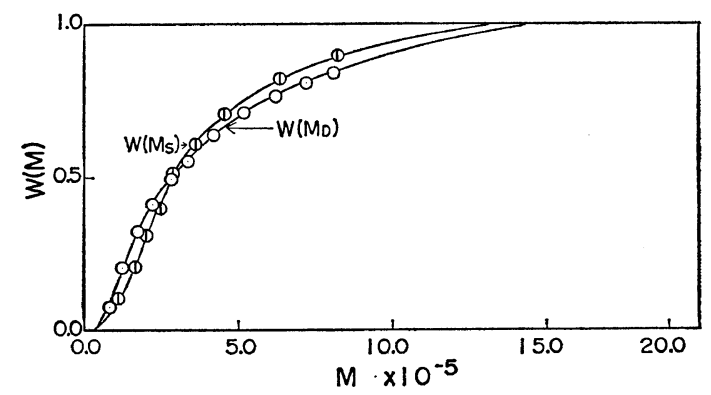

Figure 10. Integral weight distribution curues of molecular weight of PSL (linear polystyrene).

\begin{tabular}{|c|c|c|c|c|c|c|}
\hline \multirow{3}{*}{ Sample } & \multicolumn{4}{|c|}{ Molecular weight } & \multicolumn{2}{|c|}{ Branching parameter } \\
\hline & \multicolumn{2}{|c|}{ Calcd } & \multicolumn{2}{|c|}{ Obsd } & \multirow{2}{*}{$\frac{\text { Calcd }}{\left(h_{w}\right)^{3} \mathrm{~b}}$} & \multirow{2}{*}{$\frac{\text { Obsd }}{[\eta]_{\mathrm{b} \theta} /[\eta]_{1 \theta}}$} \\
\hline & $M_{w} \times 10^{-4}$ & $M_{n} \times 10^{-4}$ & $M_{w} \times 10^{-4}$ & $M_{n} \times 10^{-4}$ & & \\
\hline PSB-1 & 35.0 & 21.1 & 31.3 & 22.7 & 0.78 & 0.83 \\
\hline PSB-2 & 116.0 & 74.2 & 120.4 & 67.9 & 0.64 & 0.65 \\
\hline PSL ${ }^{\text {a }}$ & 32.8 & 24.3 & 27.0 & 20.2 & & \\
\hline
\end{tabular}

Table II. Values of molecular weights and branching parameters

a Linear polystyrene.

b $\left(h_{w}\right)^{3}=\left(\sum_{\mathrm{i}} h_{\mathrm{i}} w_{\mathrm{i}} / \sum_{\mathrm{i}} w_{\mathrm{i}}\right)^{3}$. 


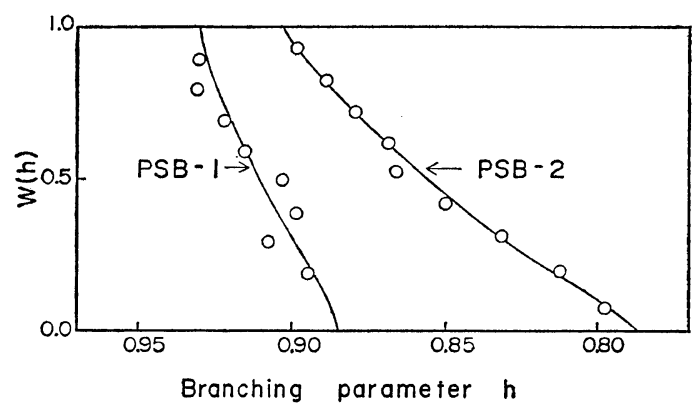

Figure 11. Integral distribution curves of branching parameter.

the intrinsic viscosities of linear and branched polymers in cyclohexane at $35^{\circ} \mathrm{C}$, respectively. The calculated values of $M_{w}, M_{n}$, and $\left(h_{w}\right)^{3}$ are in close agreement with those obtained by experiment. However, $\left(h_{w}\right)^{3}$ in Table II is not always equal to the value of $[\eta]_{b} /[\eta]_{1}$. As the value of intrinsic viscosity is a weight average value, we here assumed, following Stockmayer and Fixman, ${ }^{4}$ that the cube of the weight average branching parameter is equal to the value of $[\eta]_{b} /[\eta]_{1}$. It must now be discussed whether such a treatment is valid or not.

\section{DISCUSSION}

We have shown that the new method is experimentally simple and useful for obtaining the molecular weight and branching parameter distribution curves separately. Our method, however, involved four hypotheses. The first hypothesis is as follows. All experiments have to be carried out at the $\theta$ point, and the $\theta$-point of the branched polymer must be close to that of the corresponding linear polymer. Though our results show that the $\theta$ temperature of a branched polymer is little affected by the branching degree and is close to that of the linear polymer, polymers having the $\theta$-temperature near the room temperature are rare. Therefore, our method must be extended for good solvent systems, such as polystyrene in MEK. The second hypothesis is that the partial specific volume of the branched polymer is equal to that of the linear polymer. Our experimental results show that this seems to be valid, but this hypothesis should be experimentally con- firmed. The third hypothesis is that the correction of the pressure effect on the sedimentation constant was neglected in our experiments. According to Fujita, the value of the correction is not negligible in an organic solvent system. ${ }^{24}$ However, as a first approximation we neglected it in this work. This correction must be considered in the further development of this method. The last hypothesis is the weakest point of our method. According to the previous workers, ${ }^{18,20}$ when the molecular weight distribution curve of a polymer is measured by the diffusion method, the molecular weight distribution function or distribution function of the diffusion constant should be assumed to be analytical. In this paper, Gralén's distribution function has been assumed for the diffusion constant distribution. ${ }^{20}$ If the distribution function is inadequate for the polymer, our result may not be valid. This problem is correlated to the applicable limitation of this new method. We cannot apply the method to a sample with two peaks in the differentical distribution curve of molecular weight. Moreover, from the simple considerations of Figure 1 and eq 11 and 17, even if the sample should have one peak in the molecular weight distribution curve, the application of our method may be limited by the relation

$$
M_{\mathrm{j}} / M_{\mathrm{i}}>\left(h_{\mathrm{i}} / h_{\mathrm{j}}\right)^{2}>1
$$

where, $M_{\mathrm{i}}, M_{\mathrm{j}}, h_{\mathrm{i}}$, and $h_{\mathrm{j}}$ are molecular weights and branching parameters of molecular species of $i$ and $j$, respectively. Therefore our method is only applicable to a randomly branched polymer which has one peak in the molecular weight distribution curve and satisfies relation 21 . Then, this method has to be applied by considering the polymerization conditions and relation 21 . From the polymerization conditions and the values in Table II, the samples used in this work seem to satisfy the relation 21 . However, only two samples have been studied here. This problem also should be discussed theoretically and experimentally in further studies, and our method should be compared with the results obtained by combining GPC and sedimentation velocity measurements or GPC and viscosity measurements. Moreover, from Figures $6,7,8$, and 9 , the sedimentation constant- 
molecular weight and diffusion constant-molecular weight relationships of a branched polymer can be experimentally obtained without any effect of molecular weight distribution. This problem shall be reported in a subsequent paper.

Acknowledgment. We wish to thank Professors Takahide Saito and Naomichi Iso of Tokyo Suisan University for permission to use the Ultracentrifuge in their laboratory. Thanks are due to Professor Masatami Takeda of Tokyo College of Science for supplying a linear polystyrene sample and to Professor Taiichi Shibuya of the Faculty of Textile Science, Shinshu University, for reading and correcting the manuscript.

\section{REFERENCES}

1. C. D. Thurmond and B. H. Zimm, J. Polym. Sci., 8, 477 (1952).

2. J. E. Guillet, ibid., Part A, 1, 2869 (1963).

3. J. Blachford and R. F. Robertson, ibid., Part A, 3, 1289 (1965).

4. W. H. Stockmayer and M. Fixman, Ann. N.Y. Acad. Sci., 57, 334 (1953).

5. M. Kurata and M. Fukatsu, J. Chem. Phys., 41, 2934 (1964).

6. G. C. Berry and T. A. Orofino, ibid., 40, 1614 (1964).

7. R. W. Kilb, J. Polym. Sci., 38, 403 (1959).

8. M. Kurata, M. Abe, M. Iwama, and $M$. Matsushima, Polymer J., 3, 729 (1972).

9. K. Kamada and H. Sato, ibid., 2, 489 (1971).
10. K. Kamada and H. Sato, ibid., 2, 593 (1971).

11. E. E. Drott and R. A. Mendelson, J. Polym. Sci., Part A-2, 8, 1361 (1970).

12. E. E. Drott and R. A. Mendelson, ibid., Part A-2, 8, 1373 (1970).

13. M. Kurata, H. Okamoto, M. Iwama, M. Abe, and T. Honma, Polymer, J., 3, 739 (1972).

14. L. H. Tung, J. Polym. Sci., Part A-2, 7, 47 (1969).

15. L. H. Tung, ibid., Part A-2, 9, 759 (1971).

16. The Svedberg, "Theory of Sedimentation," in "The Ultracentrifuge," The Svedberg and K.-O. Pedersen Ed., Clarendon Press, Oxford, 1940, p 5.

17. H. W. McComic, "Sedimentation," in "Polymer Fractionation," M. J.R. Cantow, Ed., Academic Press, New York, N.Y., 1967, P 251.

18. W. Burchard and H.-J. Cantow, "Isothermal Diffusion," in "Polymer Fractionation," M. J.R. Cantow, Ed., Academic Press, New York, N.Y., 1967, p 285.

19. P. J. Flory, "Principles of Polymer Chemistry", Cornell University Press, Ithaca, New York, N.Y., 1953 p 541.

20. N. Gralén, Kolloid-Z. 95, 188 (1941).

21. H. Matsuda, M. Takeshima, S. Shimizu, and S. Kuroiwa, Makromol. Chem., 134, 309 (1970).

22. L. H. Tung and J. R. Runyon, J. Appl. Polymer Sci., 17, 1589 (1973).

23. A. Kotera, T. Saito, H. Matsuda, and K. Takemura, Rept. Progr. Polymer Phys. Japan, 14, 39 (1971).

24. H. Fujita, "Foundations of Ultra-Centrifugal Analysis," Wiley-Interscience, New York, N.Y., 1975, p 127. 